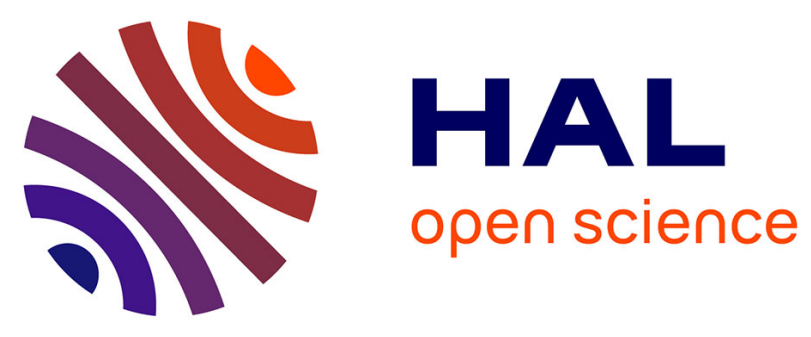

\title{
A constitutive multiphysics modeling for nearly incompressible dissipative materials: application to thermo-chemo-mechanical aging of rubbers
}

Stéphane Lejeunes, Dominique Eyheramendy, Adnane Boukamel, Alexis Delattre, Stéphane Méo, Komla Dela Ahose

\section{To cite this version:}

Stéphane Lejeunes, Dominique Eyheramendy, Adnane Boukamel, Alexis Delattre, Stéphane Méo, et al.. A constitutive multiphysics modeling for nearly incompressible dissipative materials: application to thermo-chemo-mechanical aging of rubbers. Mechanics of Time-Dependent Materials, 2018, 22 (1), pp.51-66. 10.1007/s11043-017-9351-2 . hal-01569924

\section{HAL Id: hal-01569924 https://hal.science/hal-01569924}

Submitted on 1 Jan 2018

HAL is a multi-disciplinary open access archive for the deposit and dissemination of scientific research documents, whether they are published or not. The documents may come from teaching and research institutions in France or abroad, or from public or private research centers.
L'archive ouverte pluridisciplinaire HAL, est destinée au dépôt et à la diffusion de documents scientifiques de niveau recherche, publiés ou non, émanant des établissements d'enseignement et de recherche français ou étrangers, des laboratoires publics ou privés. 


\title{
A constitutive multiphysics modeling for nearly incompressible dissipative materials: application to thermo-chemo-mechanical aging of rubbers
}

\author{
S. Lejeunes ${ }^{1}$ - D. Eyheramendy ${ }^{1} \cdot$ A. \\ Boukamel $^{2}$. A. Delattre ${ }^{3}$. S. Méo ${ }^{4}$. \\ K.D. Ahose ${ }^{1}$
}

Received: date / Accepted: date

\begin{abstract}
In this paper we investigate the modeling of chemo-physical evolution due to thermo-mechanical loadings at finite strain in soft materials. In particular we discus the question of a proper and consistent thermodynamical formulation in the case of nearly incompressible materials. The objective of this phenomenological modeling is to represent the thermo-chemo-mechanical aging that occurs in filled rubber during high cycles fatigue for some specific loading conditions.
\end{abstract}

Keywords chemo-physical aging $\cdot$ filled rubber $\cdot$ self heating

\section{Introduction}

The question of the relative influence between local chemo-physical evolutions and the local thermo-mechanical state is fundamental for materials science. This question appears for different materials and applications, e.g. material processing, environmental aging, recycling. A wide literature on these topics especially regarding experimental aspects exist. The constitutive and the numerical modeling of these phenomena have received growing attention since the last decade and one can find different approaches and different applications in the litterature. For instance, in Loeffel and Anand (2011), the authors investigate a multiphysics model of the oxydation of metallic thermal barrier coatings in severe operating conditions. In Gigliotti and Grandidier (2010), Gigliotti et al (2011) and Johlitz and 1

LMA, CNRS, UPR 7051, Centrale Marseille, Aix-Marseille Univ, F-13402 Marseille Cedex 20, France

Tel.: +33484525 597

E-mail: lejeunes@lma.cnrs-mrs.fr

IRT Raylenium, LAMIH, Univ. Valenciennes, F-59300 Famars, France

Airbus Helicopters, Marignane, France

LMR, EA 2640, Université F. Rabelais de Tours, France 
Lion (2013) the thermo-oxydation aging of a polymer matrix including chemical reactions, diffusion and mechanical coupling is proposed. The modeling of the curing of polymers and the vulcanization of rubbers, including chemo-thermo-mechanical couplings has been investigated in Lion and Höfer (2007), Mahnken (2013), Kannan and Rajagopal (2011) and André and Wriggers (2005). All these works share the same philosophy that chemo-physical evolutions are strongly coupled to the thermo-mechanical behavior and must be taken into account in a robust and consistent thermodynamical framework.

From a general scientific point of view, assuming both, a stochiometric chemical process and a local mixture as a whole hypothesis (i.e., at a material point $\mathbf{x}(\mathbf{X}, t)$ the relative velocities of chemical species are null), the question of coupling thermo-chemo-mechanical mechanisms in a continuum framework does not pose major difficulties. The appropriate thermodynamical framework can be found in Prigogine (1968) for instance. Nevertheless, even for simple cases, some questions remain opened for the case of nearly-incompressible materials. The nearlyincompressibility constraint and the fact that soft materials are sensitive to thermal dilation and chemical volume variations raises the question of the influence of chemical reaction on the thermal and mechanical states. The role of the hydrostatic pressure on the chemical process also need to be taken into account. In this paper we propose to define a mechanical volume variation directly linked to the nearly incompressibility constraint. Another question concerns the chemical contribution to the free energy. In NGuyen et al (2016), we have proposed a chemical thermodynamic free energy potential that introduces an induction temperature below which no chemical reactions occurs. This is compatible with the existence of a reference state (that is stress and evolution free). The chemical thermodynamical force (i.e. the chemical affinity) derives from the thermodynamic potential and takes into account a mechanical contribution.

In this paper, we propose to use a mixed free energy form inspired by a partial Legendre transform of the Helmoltz free energy where the volume variation is replaced by the hydrostatic pressure (as similarly adopted in Lion et al (2014)). The chemical affinity is therefore directly linked to the hydrostatic pressure. Furthermore, we investigate the case of fully incompressible materials as a limit of quasi-incompressibility. We show that the proposed model can phenomenologically represent aging phenomena that are strongly coupled to the thermal and mechanical state on trial examples.

\section{Experimental motivations}

The developments proposed in this paper are motivated by experimental observations done during studies of the fatigue life of different rubber materials: a silicone rubber filled with silica and a butadiene rubber filled with carbon black (see Grandcoin et al (2014); Delattre et al (2016)). We observe different behaviors depending both on the mechanical loading and the kind of specimens used. For thin specimens (used for tension), we mainly observed damage due to fatigue. For larger specimens, where heat build can lead to important thermal gradients in the specimen, we observe a stiffening phenomena. Obviously, this phenomena is 


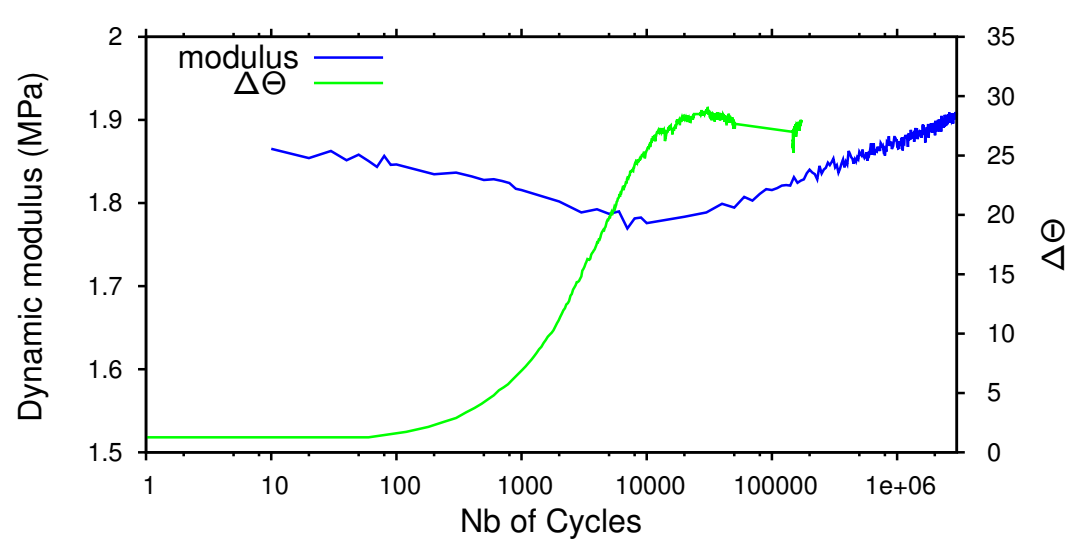

Fig. 1 Evolution of the dynamical stiffness and the surface temperature variation of a dumbell specimen submitted to fatigue cyclic test, $12 \mathrm{~Hz}, \pm 10 \%$ of dynamic amplitude with $+50 \%$ of static preload (butadiene rubber filled with carbon black).

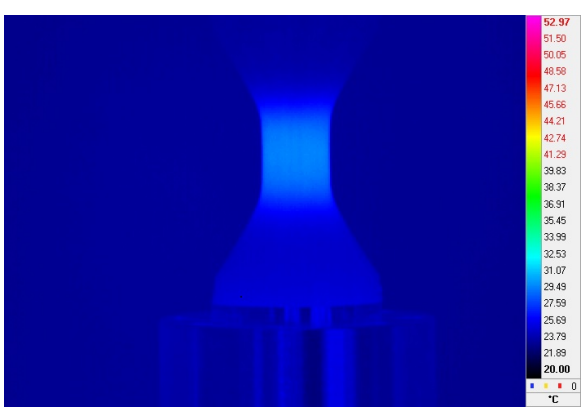

(a) 720 cycles

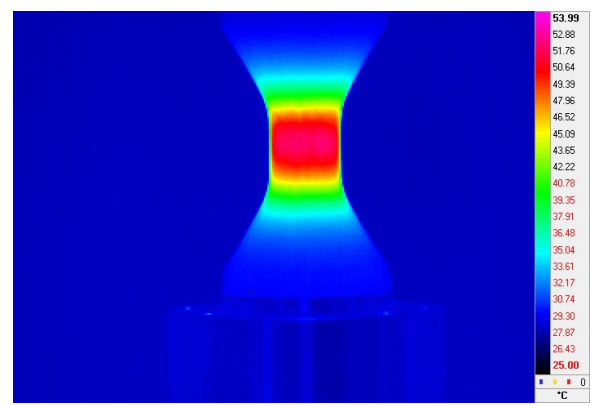

(b) 10800 cycles

Fig. 2 Temperature variation fields at the surface of a dumbell specimen during a fatigue test, $12 \mathrm{~Hz}, \pm 10 \%$ of dynamic amplitude with $+50 \%$ of static preload (butadiene rubber filled with carbon black).

strongly related to the amplitude of the mechanical loading: for high amplitudes, damage is the dominant phenomenon, and for small amplitudes behavior does not evolve.

Figure 1 shows the evolution of the dynamical stiffness and the maximum surface temperature of a filled rubber specimen during fatigue test at room temperature. These results show that for the first 10000 cycles the specimen temperature increases due to self heating while in the same time we can observe a thermal softening phenomena. This is classical for filled rubber. This softening phase may also include internal damage. After 10000 cycles, a thermal equilibrium is reached and the dynamical stiffness increases. This phenomenon may be caused by a chemo-physical evolution of the material. In figure 2 the temperature variation is heterogeneous except in the central zone of the surface of the specimen where it is nearly-homogenous. A temperature elevation of $30^{\circ}$ is observed at the surface of the specimen. The temperature elevation is probably higher inside the specimen. 


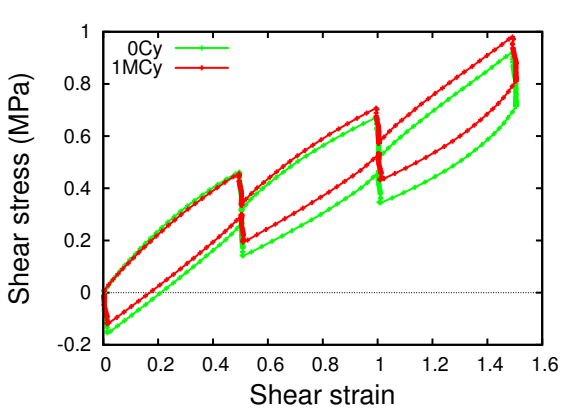

(a) Steps relaxation test at room temperature on the same specimen after fatigue

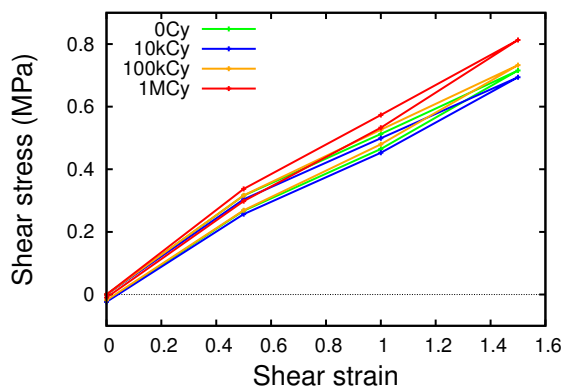

(b) Ends of relaxation tests at room temperature on the same specimen after fatigue

Fig. 3 Mechanical characterization tests at room temperature on double shear specimens that are previously submitted to fatigue $(12 \mathrm{~Hz}, \pm 50 \%$ of dynamical amplitude)

Aging can be confirmed with mechanical characterization test lead after fatigue at room temperature. In figure 3 , we show simple shear relaxation tests on a double-shear specimens that are previously submitted to fatigue. We observe a stiffer relaxed behavior after fatigue. Obviously, this permanent (irreversible) stiffing or aging phenomena is only observed for specific fatigue conditions on large enough specimens. More details on the experimental protocol adopted for fatigue and characterisation tests can be found in Grandcoin et al (2014). In Garnier et al (2013), the authors also observed a non monotonous evolution of the dynamical stiffness during cyclic loading tests with an unexpected stiffening effect on a filled nitrile rubber.

\section{Constitutive modeling}

\subsection{Kinematic}

We adopte the same approach as in NGuyen et al (2016). We consider a chemical process with a single stochiometric reaction. We can define a local internal variable $\xi(\mathbf{X}, t)$ that describes the relative advance of reaction (or chemical conversion). We assume that both the mechanical and the thermal behavior are related to the chemical process through the chemical conversion $\xi$. Typically $\xi$, can be taken as the normalized concentration of newly created sulfur cross-links at a material point.

We also assume that, in the reference state, no chemical reaction occurs and the material is stress free. As we are interested in a thermal aging phenomena, we also assume that the evolution and the initiation of the chemical reaction are strongly dependent on the temperature. Thus, we assume it exists an initiation temperature under which no reactive process can occur. To consider the nearly incompressible behavior, the thermal dilatation, the chemical volume variation and the viscoelastic strain, it is adopted the following splitting of the deformation gradient:

$$
\mathbf{F}=J^{1 / 3} \overline{\mathbf{F}}_{e} \cdot \overline{\mathbf{F}}_{v} \quad \text { with } \quad J=J_{\Theta} J_{m} J_{\xi}
$$


where $\overline{\mathbf{F}}_{e}$ and $\overline{\mathbf{F}}_{v}$ are isochoric elastic and viscous deformation gradients. The thermal, mechanical and chemical volume variation are given respectively by:

$$
\begin{aligned}
J_{\Theta} & =1+\alpha_{\Theta}\left(\Theta-\Theta_{0}\right) \\
J_{m} & =\frac{J}{J_{\Theta} J_{\xi}} \\
J_{\xi} & =1+\alpha_{\xi} g(\xi)
\end{aligned}
$$

where $\alpha_{\Theta}$ and $\alpha_{\xi}$ are material coefficients of thermal dilatation and chemical shrinkage/dilation ${ }^{1}, \Theta_{0}$ is the initial (reference) temperature, $g(\xi)$ is a chemical shrinkage/dilatation function.

\subsection{Thermodynamic}

In the current configuration, the first and second thermodynamic principles (conservation of energy and entropy production) take the following local form (the dot superscript refers as the so-called material time derivative):

$$
\begin{gathered}
\rho \dot{e}-\boldsymbol{\sigma}: \mathbf{D}-\rho r+\operatorname{div}_{\boldsymbol{x}} \mathbf{q}=0 \\
\rho \Theta \dot{s}-\rho r+\operatorname{div}_{\boldsymbol{x}} \mathbf{q}-\frac{\operatorname{grad}_{\boldsymbol{x}} \Theta}{\Theta} \cdot \mathbf{q} \geq 0
\end{gathered}
$$

where $e$ is the specific internal energy, $\boldsymbol{\sigma}$ is the Cauchy stress, $r$ is a furnished (exterior) heat power (defined by volume unit) and $\mathbf{q}$ is the eulerian heat flux, $s$ is the specific entropy, $\rho$ is the current density, $\operatorname{div}_{\boldsymbol{x}}$ and $\operatorname{grad}_{\boldsymbol{x}}$ are respectively eulerian divergence and eulerian gradient. It is more practical to introduce another state potential, the free energy that takes mainly two forms: Gibbs or Helmoltz. We propose in this paper to use a mixed free energy form defined as follows:

$$
\varphi=e+\beta-\Theta s=\psi+\beta
$$

where $\psi$ is the Helmoltz free energy and $\beta$ is a potential related to the nearly incompressible behavior. The free energy $\varphi$ is a mixed free energy that depends on state variables, $\overline{\mathbf{B}}, \Theta$, on internal variables, $\overline{\mathbf{B}}_{\mathbf{e}}, \xi$, and on hydrostatic pressure $p$. The potential $\beta$ depends on both $p$ and $J$. This mixed form can be viewed as a partial Legendre transformation of the Helmoltz free energy in some simple situations. This concept has been recently used in Lion et al (2014) to investigate the caloric behavior of nearly incompressible media with irreversible volume changes.

Combining eq. (5) and eq. (6) with definition (7), one can obtain the following inequality:

$$
\phi=\underbrace{\boldsymbol{\sigma}: \mathbf{D}-\rho \dot{\varphi}+\rho \dot{\beta}-\rho \dot{\Theta} s}_{\phi_{\text {intr }}}-\underbrace{\frac{\operatorname{grad}_{x} \Theta}{\Theta} \cdot \mathbf{q}}_{\phi_{\Theta}} \geq 0
$$

where $\phi$ is the total dissipation $\phi_{i n t r}$ and $\phi_{\Theta}$ are respectively the intrinsic and thermal parts of the dissipation. The material time derivatives of kinetic variables

\footnotetext{
1 Depending on the process considered, chemical evolution can lead to shrinkage or expansion.
} 
are defined from the following expressions:

$$
\begin{aligned}
\dot{J} & =J(\mathbf{1}: \mathbf{D}) \\
\dot{\overline{\mathbf{B}}} & =\mathbf{L} \cdot \overline{\mathbf{B}}+\overline{\mathbf{B}} \cdot \mathbf{L}^{\mathrm{T}}-\frac{2}{3}(\mathbf{1}: \mathbf{D}) \overline{\mathbf{B}} \\
\dot{\overline{\mathbf{B}}}_{\mathbf{e}} & =\mathbf{L} \cdot \overline{\mathbf{B}}_{\mathbf{e}}+\overline{\mathbf{B}}_{\mathbf{e}} \cdot \mathbf{L}^{\mathrm{T}}-2 \overline{\mathbf{V}}_{\mathbf{e}} \cdot \overline{\mathbf{D}}_{\mathbf{v}}^{\mathbf{o}} \cdot \overline{\mathbf{V}}_{\mathbf{e}}-\frac{2}{3}(\mathbf{1}: \mathbf{D}) \overline{\mathbf{B}}_{\mathbf{e}}
\end{aligned}
$$

where $\overline{\mathbf{B}}=\overline{\mathbf{F}} \cdot \overline{\mathbf{F}}^{\mathrm{T}}$ and $\overline{\mathbf{B}}_{\mathbf{e}}=\overline{\mathbf{F}}_{\mathbf{e}} \cdot \overline{\mathbf{F}}_{\mathbf{e}}^{\mathrm{T}}$ are isochoric left Cauchy-Green tensors, $\overline{\mathbf{V}}_{\mathbf{e}}$ comes from the polar decomposition $\overline{\mathbf{F}}_{\mathbf{e}}=\overline{\mathbf{V}}_{\mathbf{e}} \cdot \overline{\mathbf{R}}_{\mathbf{e}}, \overline{\mathbf{D}}_{\mathbf{v}}^{\mathbf{o}}=\overline{\mathbf{R}}_{\mathbf{e}} \cdot \mathbf{D}_{v} \cdot \overline{\mathbf{R}}_{\mathbf{e}}^{\mathrm{T}}$. Assuming the following dependence: $\varphi\left(\overline{\mathbf{B}}, \overline{\mathbf{B}}_{\mathbf{e}}, \Theta, \xi, p\right)$ and $\beta(J, p, \Theta, \xi)$, The material time derivative of $\varphi$ and $\beta$ can be expressed as:

$$
\begin{aligned}
\dot{\varphi}= & \left(2 \overline{\mathbf{B}} \cdot \frac{\partial \varphi}{\partial \overline{\mathbf{B}}}\right)^{\mathrm{D}}: \mathbf{D}+\left(2 \overline{\mathbf{B}}_{\mathbf{e}} \cdot \frac{\partial \varphi}{\partial \overline{\mathbf{B}}_{\mathbf{e}}}\right)^{\mathrm{D}}: \mathbf{D}+\frac{\partial \varphi}{\partial \Theta} \dot{\Theta}+\frac{\partial \varphi}{\partial \xi} \dot{\xi}+\frac{\partial \varphi}{\partial p} \dot{p} \\
& -\left(2 \overline{\mathbf{B}}_{\mathbf{e}} \cdot \frac{\partial \varphi}{\partial \overline{\mathbf{B}}_{\mathbf{e}}}\right): \overline{\mathbf{D}}_{\mathbf{v}}^{\text {o }} \\
\dot{\beta}= & J \frac{\partial \beta}{\partial J} \mathbf{1}: \mathbf{D}+\frac{\partial \Theta}{\partial \Theta} \dot{\Theta}+\frac{\partial p}{\partial p} \dot{p}+\frac{\partial \xi}{\partial \xi} \dot{\xi}
\end{aligned}
$$

Inserting eq. 12 and 13 in 8 , the dissipation can be rewritten as:

$$
\begin{aligned}
\phi & =\left(\boldsymbol{\sigma}-2 \rho\left(\overline{\mathbf{B}} \cdot \frac{\partial \varphi}{\partial \overline{\mathbf{B}}}\right)^{\mathrm{D}}-2 \rho\left(\overline{\mathbf{B}}_{\mathbf{e}} \cdot \frac{\partial \varphi}{\partial \overline{\mathbf{B}}_{\mathbf{e}}}\right)^{\mathrm{D}}+\rho J \frac{\partial \beta}{\partial J} \mathbf{1}\right): \mathbf{D}+\rho\left(-s-\frac{\partial \varphi}{\partial \Theta}+\frac{\partial \beta}{\partial \Theta}\right) \dot{\Theta} \\
& +\rho\left(\frac{\partial \beta}{\partial p}-\frac{\partial \varphi}{\partial p}\right) \dot{p}+\rho\left(\frac{\partial \beta}{\partial \xi}-\frac{\partial \varphi}{\partial \xi}\right) \dot{\xi}+\left(2 \rho \overline{\mathbf{B}}_{\mathbf{e}} \cdot \frac{\partial \varphi}{\partial \overline{\mathbf{B}}_{\mathbf{e}}}\right): \overline{\mathbf{D}}_{\mathbf{v}}^{\mathbf{o}} \\
& +\left(-\frac{\operatorname{grad}_{\boldsymbol{x}} \Theta}{\Theta}\right) \cdot \mathbf{q} \geq 0
\end{aligned}
$$

We assume that dissipation is only due to both thermal diffusion and internal variable evolutions ${ }^{2}$, the following constitutive equations are obtained:

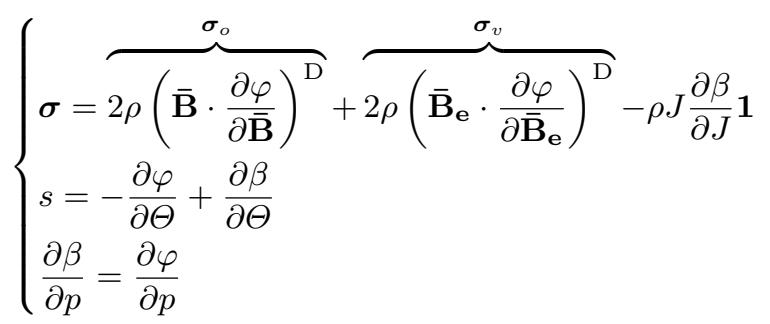

and the following inequalities must holds:

$$
\left\{\begin{array}{l}
\phi_{m}=\left(2 \rho \overline{\mathbf{B}}_{\mathbf{e}} \cdot \frac{\partial \varphi}{\partial \overline{\mathbf{B}}_{\mathbf{e}}}\right): \overline{\mathbf{D}}_{\mathbf{v}}^{\mathbf{o}} \geq 0 \quad \forall \overline{\mathbf{D}}_{\mathbf{v}}^{\mathbf{o}} \\
\phi_{\xi}=\rho\left(\frac{\partial \beta}{\partial \xi}-\frac{\partial \varphi}{\partial \xi}\right) \dot{\xi} \geq 0 \quad \forall \dot{\xi} \\
\phi_{\Theta}=\left(-\frac{\operatorname{grad}_{\boldsymbol{x}} \Theta}{\Theta}\right) \cdot \mathbf{q} \geq 0 \quad \forall \mathbf{q}
\end{array}\right.
$$

\footnotetext{
${ }^{2}$ Internal variables evolutions are assumed to be independent from each others.
} 
Using the definition of intrinsic dissipation, the energy conservation, eq. (5) can be re-written as follows:

$$
\rho \Theta \dot{s}=\underbrace{\phi_{\text {intr }}}_{\phi_{m}+\phi_{\xi}}+\rho r-\operatorname{div}_{\mathbf{x}} \mathbf{q}
$$

From the constitutive equation of the entropy given at eq. (15), the material time derivative of the entropy can be derived and inserted in the previous equation:

$$
\rho C_{p} \dot{\Theta}=\phi_{m}+\phi_{\xi}+l_{m}+l_{\xi}+\rho r-\operatorname{div}_{\mathbf{x}} \mathbf{q}
$$

where $l_{m}, l_{\xi}$ are respectively mechanical and chemical coupling terms defined by:

$$
\begin{aligned}
l_{m} & =\Theta\left(\frac{\partial \boldsymbol{\sigma}}{\partial \Theta}: \mathbf{D}-\frac{\partial \boldsymbol{\sigma}_{v}}{\partial \Theta}: \overline{\mathbf{D}}_{\mathbf{v}}^{\mathbf{o}}\right)+\rho \Theta\left(\frac{\partial^{2} \varphi}{\partial \Theta \partial p}-\frac{\partial^{2} \beta}{\partial \Theta \partial p}\right) \dot{p} \\
l_{\xi} & =\rho \Theta\left(\frac{\partial^{2} \varphi}{\partial \Theta \partial \xi}-\frac{\partial^{2} \beta}{\partial \Theta \partial \xi}\right) \dot{\xi}
\end{aligned}
$$

The heat capacity is defined as follows:

$$
C_{p}=-\Theta\left(\frac{\partial^{2} \varphi}{\partial \Theta^{2}}-\frac{\partial^{2} \beta}{\partial \Theta^{2}}\right)
$$

\subsection{A simple thermo-chemo-viscoelastic model}

We consider the case of isotropic behaviors (thermal and mechanical) and we propose to adopt the following potentials:

$$
\begin{aligned}
\rho_{0} \varphi= & \mu_{0}\left(I_{1}(\overline{\mathbf{B}})-3\right)+\mu_{v}\left(I_{1}\left(\overline{\mathbf{B}}_{\mathbf{e}}\right)-3\right)+C_{0}\left(\Theta-\Theta_{0}-\Theta \log \left(\frac{\Theta}{\Theta_{0}}\right)\right) \\
& -C_{1} \frac{\left(\Theta-\Theta_{0}\right)^{2}}{2 \Theta_{0}}+C_{2}\left(\Theta_{i n d} \log \left(\frac{\Theta}{\Theta_{i n d}}\right) \frac{(1-\xi)^{n+1}}{n+1}\right. \\
& \left.-\Theta_{0} \log \left(\frac{\Theta_{0}}{\Theta_{i n d}}\right)\right) \\
\rho_{0} \beta= & p\left(J_{m}-1\right)+\frac{p^{2} J_{\Theta} J_{\xi}}{2 K_{v}}
\end{aligned}
$$

where $I_{1}(\overline{\mathbf{B}}), I_{1}\left(\overline{\mathbf{B}}_{\mathbf{e}}\right)$ are the first invariants of the left Cauchy-Green total and elastic isochoric tensors, $\mu_{0}, \mu_{v}$ are the mechanical modulus that depend on $\Theta$ and $\xi, C_{0}$ and $C_{1}$ are the heat coefficients (that are assumed to be constant in this paper), $C_{2}$ is a heat coefficient related to the chemical process (that is exothermic in the present case), $\Theta_{\text {ind }}$ is an induction temperature for the chemical process ${ }^{3}$, $n$ is a chemical parameter, $K_{v}$ is the compressibility modulus (that is assumed as constant). The initial mass density $\rho_{0}$ is related to the current mass density through the relation: $\rho=J \rho_{0}$.

We can remark that the mixed potential $\beta$ is similar to a perturbed Lagrangian form used in the case of purely incompressible formulation for which $K v \rightarrow \infty$.

\footnotetext{
3 For free hydrostatic pressure conditions, no chemical reactions are assumed to occurs if $\Theta<\Theta_{\text {ind }}$
} 
In this case $p$ can be viewed as a Lagrange multiplier. We can also remark that the nearly incompressible mechanical behavior strictly act on $J_{m}$ and not on $J$. Therefore, the modulus $K_{v}$ has clearly the sense of a mechanical compressibility modulus.

Using definitions 22 and 23 in 15 , we obtain the following:

$$
\begin{aligned}
\boldsymbol{\sigma}= & \frac{2}{J} \mu_{0}(\Theta, \xi) \overline{\mathbf{B}}^{\mathrm{D}}+\frac{2}{J} \mu_{v}(\Theta, \xi) \overline{\mathbf{B}}_{\mathbf{e}}^{\mathrm{D}}-\frac{p}{J_{\Theta} J_{\xi}} \mathbf{1} \\
s= & -\frac{1}{\rho_{0}} \frac{\partial \mu_{0}}{\partial \Theta}\left(I_{1}(\overline{\mathbf{B}})-3\right)-\frac{1}{\rho_{0}} \frac{\partial \mu_{v}}{\partial \Theta}\left(I_{1}\left(\overline{\mathbf{B}}_{\mathbf{e}}\right)-3\right)+\frac{C_{0}}{\rho_{0}} \log \left(\frac{\Theta}{\Theta_{0}}\right) \\
& +\frac{C_{1}}{\rho_{0}} \frac{\left(\Theta-\Theta_{0}\right)}{\Theta_{0}}-\frac{C_{2}}{\rho_{0}} \frac{\Theta_{\text {ind }}}{\Theta} \frac{(1-\xi)^{n+1}}{n+1}-\frac{p \alpha_{\Theta} J}{J_{\Theta}^{2} J_{\xi}}+\frac{p^{2} \alpha_{\Theta} J_{\xi}}{2 K_{v}} \\
p= & -\frac{K_{v}}{J_{\Theta} J_{\xi}}\left(J_{m}-1\right)
\end{aligned}
$$

The heat capacity and latent heat terms are obtained from eqs. (21), (19) and (20):

$$
\begin{aligned}
C_{p}= & -\frac{\Theta}{\rho_{0}} \frac{\partial^{2} \mu_{0}}{\partial \Theta^{2}}\left(I_{1}(\overline{\mathbf{B}})-3\right)-\frac{\Theta}{\rho_{0}} \frac{\partial^{2} \mu_{v}}{\partial \Theta^{2}}\left(I_{1}\left(\overline{\mathbf{B}}_{\mathbf{e}}\right)-3\right)+\frac{C_{0}}{\rho_{0}} \\
& +\frac{C_{1}}{\rho_{0}} \frac{\Theta}{\Theta_{0}}+\frac{C_{2}}{\rho_{0}} \frac{\Theta_{\text {ind }}}{\Theta} \frac{(1-\xi)^{n+1}}{n+1}+\frac{2}{\rho_{0}} \frac{\Theta p \alpha_{\Theta}^{2} J}{J_{\Theta}^{3} J_{\xi}} \\
l_{m}= & \Theta\left(\frac{\partial \boldsymbol{\sigma}}{\partial \Theta}: \mathbf{D}-\frac{\partial \boldsymbol{\sigma}_{v}}{\partial \Theta}: \overline{\mathbf{D}}_{\mathbf{v}}^{\mathbf{o}}\right)+\frac{\Theta}{J}\left(\frac{J_{\alpha_{\Theta}}}{J_{\Theta}^{2} J_{\xi}}-\frac{p \alpha_{\Theta} J_{\xi}}{K_{v}}\right) \dot{p} \\
l_{\xi}= & \frac{\Theta}{J}\left(\frac{\partial^{2} \mu_{0}}{\partial \Theta \partial \xi}\left(I_{1}(\overline{\mathbf{B}})-3\right)+\frac{\partial^{2} \mu_{v}}{\partial \Theta \partial \xi}\left(I_{1}\left(\overline{\mathbf{B}}_{\mathbf{e}}\right)-3\right)\right) \dot{\xi} \\
& -\frac{C_{2} \Theta_{i n d}}{J}(1-\xi)^{n} \dot{\xi}+\frac{\Theta}{J} \frac{\partial g}{\partial \xi}\left(-\frac{p J_{\alpha_{\Theta} \alpha_{\xi}}}{J_{\Theta}^{2} J_{\xi}^{2}}-\frac{p^{2} \alpha_{\Theta} \alpha_{\xi}}{2 K_{v}}\right) \dot{\xi}
\end{aligned}
$$

From eq (27), one can deduce that the physical requirement $C_{p}>0$ implies constraints on the material parameters and their evolutions. As we assume the independence of internal variables evolutions, we have to satisfy the following inequalities:

$$
\begin{cases}\phi_{m}=\left(\frac{2 \mu_{v}}{J} \overline{\mathbf{B}}_{\mathbf{e}}\right): \overline{\mathbf{D}}_{\mathbf{v}}^{\mathbf{o}} \geq 0 & \forall \overline{\mathbf{D}}_{\mathbf{v}}^{\mathbf{o}} \\ \phi_{\xi}=A_{\xi} \dot{\xi} \geq 0 \quad \forall \dot{\xi} & \\ \phi_{\Theta}=\left(-\frac{\operatorname{grad}_{\boldsymbol{x}} \Theta}{\Theta}\right) \cdot \mathbf{q} \geq 0 \quad \forall \mathbf{q}\end{cases}
$$

where $A_{\xi}$ is the chemical affinity defined by:

$$
\begin{aligned}
A_{\xi}= & \frac{C_{2}}{J} \Theta_{i n d} \log \left(\frac{\Theta}{\Theta_{\text {ind }}}\right)(1-\xi)^{n}-\frac{1}{J} \frac{\partial \mu_{0}}{\partial \xi}\left(I_{1}(\overline{\mathbf{B}})-3\right)-\frac{1}{J} \frac{\partial \mu_{v}}{\partial \xi}\left(I_{1}\left(\overline{\mathbf{B}}_{\mathbf{e}}\right)-3\right) \\
& +p \alpha_{\xi} \frac{\partial g}{\partial \xi}\left(\frac{-1}{J_{\Theta} J_{\xi}^{2}}+\frac{p J_{\Theta}}{2 K_{v} J}\right)
\end{aligned}
$$


One can remark that a fully coupled formulation is obtained: chemical affinity depends on temperature, hydrostatic pressure, mechanical elastic isochoric transformations. The mechanical contribution to the chemical evolution depends on the evolution of mechanical parameters with respect to chemical states and to function $g$. We propose to adopt the following evolution equations that fulfill requirements of eqs. (30) and objectivity:

$$
\left\{\begin{array}{l}
\overline{\mathbf{D}}_{\mathbf{v}}^{\mathbf{o}}=\frac{1}{2 \tau} \overline{\mathbf{B}}_{\mathbf{e}}^{\mathrm{D}} \\
\dot{\xi}=k(\Theta)<A_{\xi}> \\
\mathbf{q}=-k_{t} \operatorname{grad}_{\boldsymbol{x}} \Theta
\end{array}\right.
$$

where $\tau$ is a characteristic relaxation time (that can depend on $\Theta, \xi$ ), $k$ is a kinetic term that depends on $\Theta, k_{t}$ is an isotropic conductivity parameter which is assumed as constant in this paper. The Mac-Cauley brackets ${ }^{4},<\bullet>$ are introduced in the evolution law to avoid undesirable chemical reversion.

Finally, we assume that the chemical reaction leads to shrinkage defined from the following function:

$$
g(\xi)=\frac{\exp ^{-1}-\exp ^{-(1-\xi)^{2}}}{\exp ^{-1}}
$$

\section{Applications to homogeneous test cases}

\begin{tabular}{|c|c|c|c|}
\hline Density & $\begin{array}{c}\rho_{0}\left(K g / m^{3}\right) \\
1000\end{array}$ & & \\
\hline Thermal & $\begin{array}{c}\alpha_{\Theta}\left(K^{-1}\right) \\
2.2 e^{-4}\end{array}$ & $\begin{array}{c}C_{0}\left(J / m^{3} / K\right) \\
4.6 e^{6}\end{array}$ & $\begin{array}{c}k_{t}(W / m / K) \\
0.22\end{array}$ \\
\hline Mechanical & $\begin{array}{c}K_{v}(P a) \\
1.0 e^{9} \\
\tau(s) \\
0.0818-0.0183 \xi-0.001(\Theta-273) \\
\end{array}$ & $\begin{array}{c}\mu_{0}(P a) \\
1 . e^{6}\left(0.2+1.5 e^{-3}(\Theta-273)+0.35 \xi\right) \\
\mu_{v}(P a) \\
1 . e^{5}(0.2+2.5 \xi)+8 . e^{7} /(\Theta-200)\end{array}$ & \\
\hline Chemical & $\begin{array}{c}C_{2}\left(J / m^{3} / K\right) \\
3.0 e^{5} \\
n \\
1.3 \\
\end{array}$ & $\begin{array}{c}A\left((P a . s)^{-1}\right) \\
1.3 e^{-6} \\
\alpha \xi \\
1 . e^{-3} \\
\end{array}$ & $\begin{array}{c}\Theta_{i}(K) \\
343\end{array}$ \\
\hline
\end{tabular}

The material parameters used for the tests are given in table 1.

Table 1 Material parameters

4.1 Adiabatic cyclic simple shear with constant hydrostatic pressure

The transformation is assumed to be homogeneous and corresponds to simple shear with isotropic dilatation. The deformation gradient and the eulerian gradient of

\footnotetext{
${ }^{4}$ Mac-Cauley brackets are defined by $\langle f\rangle=f$ if $f>0$ and $\langle f\rangle=0$ otherwise.
} 
velocity are expressed as follows ${ }^{5}$ :

$$
\mathbf{F}(t)=\left[\begin{array}{ccc}
\lambda^{1 / 3} & \gamma(t) & 0 \\
0 & \lambda^{1 / 3} & 0 \\
0 & 0 & \lambda^{1 / 3}
\end{array}\right], \quad \mathbf{L}(t)=\left[\begin{array}{ccc}
0 & \gamma^{\prime}(t) / \lambda^{1 / 3} & 0 \\
0 & 0 & 0 \\
0 & 0 & 0
\end{array}\right], \quad \gamma(t)=\gamma_{0} \sin (2 \Pi f t)
$$

where $f$ is the frequency, $\gamma_{0}$ is the amplitude of the shear deformation and $\lambda$ is the dilatation. The viscoelastic Cauchy-Green tensor is assumed to be defined as follows:

$$
\overline{\mathbf{B}}_{\mathbf{e}}(t)=\left[\begin{array}{ccc}
B_{e 11}(t) & B_{e 12}(t) & 0 \\
B_{e 12}(t) & B_{e 22}(t) & 0 \\
0 & 0 & 1 /\left(B_{e 11}(t) B_{e 22}(t)-B_{e 12}(t)^{2}\right)
\end{array}\right]
$$

Using eqs. (24) and (26), the shear stress and the hydrostatic pressure can be expressed as (time dependance does not appear for the sake of simplicity):

$$
\begin{aligned}
\boldsymbol{\sigma}_{12} & =\frac{2 \mu_{0}(\Theta, \xi)}{\lambda^{4 / 3}} \gamma+\frac{2 \mu_{v}}{\lambda^{4 / 3}}(\Theta, \xi) B_{e 12} \\
p & =\frac{-K_{v}}{J_{\Theta} J_{\xi}}\left(\frac{\lambda}{J_{\Theta} J_{\xi}}-1\right)
\end{aligned}
$$

The dilatation is therefore defined by:

$$
\lambda=J_{\Theta} J_{\xi}\left(1-p \frac{J_{\Theta} J_{\xi}}{K_{v}}\right)
$$

The mechanical and chemical evolutions equations are obtained from eqs. (32) as follows:

$$
\begin{aligned}
& \dot{\overline{\mathbf{B}}}_{\mathbf{e}}=\mathbf{L} \cdot \overline{\mathbf{B}}_{\mathbf{e}}+\overline{\mathbf{B}}_{\mathbf{e}} \cdot \mathbf{L}^{\mathrm{T}}-\frac{1}{\tau(\Theta, \xi)} \overline{\mathbf{B}}_{\mathbf{e}}^{\mathrm{D}} \cdot \overline{\mathbf{B}}_{\mathbf{e}} \\
& \dot{\xi}=k(\Theta)<\frac{C_{2}}{\lambda} \Theta_{i n d} \log \left(\frac{\Theta}{\Theta_{i n d}}\right)(1-\xi)^{n}-\frac{\partial \mu_{0}}{\partial \xi} \frac{\gamma^{2}}{\lambda^{5 / 3}} \\
& -\frac{1}{\lambda} \frac{\partial \mu_{v}}{\partial \xi}\left(\operatorname{tr}\left(\overline{\mathbf{B}}_{\mathbf{e}}\right)-3\right)+p \alpha_{\xi} \frac{\partial g}{\partial \xi}\left(\frac{-1}{J_{\Theta} J_{\xi}^{2}}+\frac{p J_{\Theta}}{2 \lambda K_{v}}\right)>
\end{aligned}
$$

and the chemical kinetic term is defined from classical Arrhenius behavior:

$$
k(\Theta)=A \exp ^{-\frac{E_{a}}{R \Theta}}
$$

where $R=8.314 \mathrm{~J} / \mathrm{mol} / \mathrm{K}$ is the ideal gaz constant, $A$ is the kinetic constant and $E_{a}$ is the activation energy. The thermal equilibrium in adiabatic condition can

\footnotetext{
5 As we consider constant hydrostatic tests, the dilatation amplitude $\lambda$ do not depends explicitly on time but only on $\xi, \Theta, p$
} 
be written as:

$$
\begin{aligned}
\frac{\rho_{0}}{\lambda} C_{p} \dot{\Theta} & =l_{m}+l_{\xi}+\phi_{m}+\phi_{\xi}, \quad \Theta(0)=293 K \\
\rho_{0} C_{p} & =-\Theta \frac{\partial^{2} \mu_{v}}{\partial \Theta^{2}}\left(\operatorname{tr}\left(\overline{\mathbf{B}}_{\mathbf{e}}\right)-3\right)+C_{0} \\
& +C_{1} \frac{\Theta}{\Theta_{0}}+C_{2} \frac{\Theta_{\text {ind }}}{\Theta} \frac{(1-\xi)^{n+1}}{n+1}+2 \Theta \frac{p \alpha_{\Theta}^{2} \lambda}{J_{\Theta}^{3} J_{\xi}} \\
l_{m} & =\Theta\left(\frac{\partial \boldsymbol{\sigma}}{\partial \Theta}: \mathbf{D}-\frac{1}{2 \tau} \frac{\partial \boldsymbol{\sigma}_{v}}{\partial \Theta}: \overline{\mathbf{B}}_{\mathbf{e}}^{\mathrm{D}}\right) \\
l_{\xi} & =-\frac{C_{2} \Theta_{i n d}}{\lambda}(1-\xi)^{n} \dot{\xi}+\frac{\Theta}{\lambda} \frac{\partial g}{\partial \xi}\left(-\frac{p \lambda \alpha_{\Theta} \alpha_{\xi}}{J_{\Theta}^{2} J_{\xi}^{2}}-\frac{p^{2} \alpha_{\Theta} \alpha_{\xi}}{2 K_{v}}\right) \dot{\xi}
\end{aligned}
$$

The set of differential and algebraic equations (36), (38), (45) can be numerically solved for given values of $p$ which is assumed constant in this test and $\gamma(t)$. In this paper, we have used a monolithic forward-Euler integration scheme ${ }^{6}$.

In figure 4, the activation of a chemical evolution with a self-heating due to a cyclic mechanical loading is shown. One can remark that when the temperature increase is too small the reaction rate is strictly zero (induction phase). In figures 5 , the influence of the hydrostatic pressure on the chemical conversion and temperature evolution is shown. A compression state (positive values of $p$ ) is more favorable to a dilatation state (negative values of $p$ ). Computing the value of the dynamic shear modulus for each cycle, we can plot its evolution for various shear amplitudes. Figure 6 shows that the proposed model can reproduce the phenomena experimentally observed in figure 1 from a qualitative point of view. A thermal softening is followed by a stiffening phase. Obviously, this phenomena only occurs if self-heating is sufficient, i.e. when the mechanical amplitude is large enough.

\subsection{Adiabatic temperature aging under constant elongation}

In this example, we investigate the case of an adiabatic aging of a band of rubber under constant elongation. The mechanical behavior is assumed to be fully incompressible. The deformation gradient and the eulerian gradient of velocity are defined as follows:

$$
\begin{aligned}
& \mathbf{F}(t)=\left[\begin{array}{ccc}
\lambda(t) & 0 & 0 \\
0 & \sqrt{\frac{J_{\Theta} J_{\xi}}{\lambda(t)}} & 0 \\
0 & 0 & \sqrt{\frac{J_{\Theta} J_{\xi}}{\lambda(t)}}
\end{array}\right], \quad \mathbf{L}(t)=\left[\begin{array}{ccc}
\lambda^{\prime}(t) & 0 & 0 \\
0 & -\frac{\lambda^{\prime}(t) \sqrt{J_{\Theta} J_{\xi}}}{2 \lambda(t)^{3 / 2}} & 0 \\
0 & 0 & -\frac{\lambda^{\prime}(t) \sqrt{J_{\Theta} J_{\xi}}}{2 \lambda(t)^{3 / 2}}
\end{array}\right] \\
& \lambda(t)=\left\{\begin{array}{c}
1+\frac{\lambda_{0} t}{0.1} \text { if } t \leq 0.1 \\
1+\lambda_{0} \text { if } t>0.1
\end{array}\right.
\end{aligned}
$$

\footnotetext{
6 The explicit resolution of differential equations must be done with care on the time increment.
} 


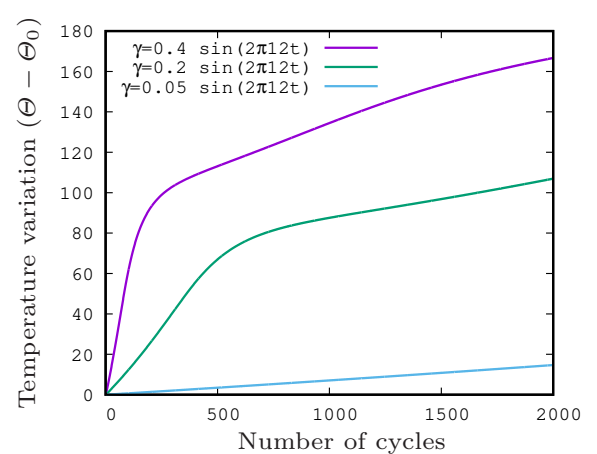

(a) Temperature according to the number of cycles

Fig. 4 Results of the cyclic adiabatic shear test for various dynamic amplitudes of shear at $f=12 \mathrm{~Hz}, \mathrm{p}=0 \mathrm{Mpa}$

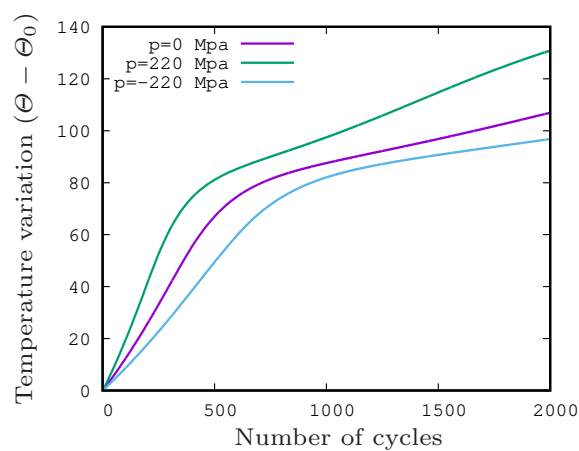

(a) Temperature upon the number of cycles

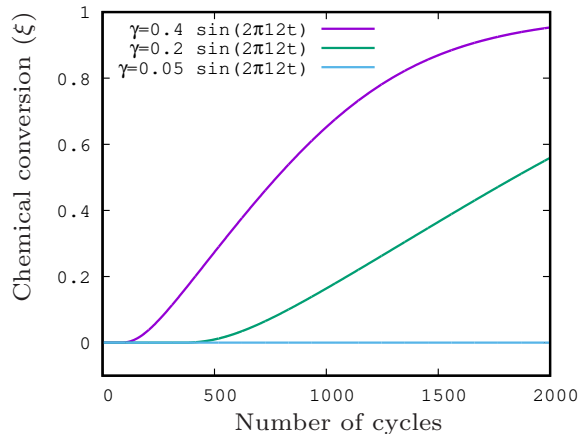

(b) Chemical conversion according to the number of cycles

Fig. 5 Results of the cyclic adiabatic shear test for various values of $p$ at $f=12 H z, \gamma_{0}=0.2$

The viscoelastic Cauchy-Green tensor is assumed to be as follows:

$$
\overline{\mathbf{B}}_{\mathbf{e}}(t)=\left[\begin{array}{ccc}
B_{e 11}(t) & 0 & 0 \\
0 & B_{e 22}(t) & 0 \\
0 & 0 & 1 /\left(B_{e 11}(t) B_{e 22}(t)\right)
\end{array}\right]
$$

Using eq. (24), the non vanishing stress components are given by:

$$
\begin{aligned}
\sigma_{11} & =\frac{4}{3} \frac{\mu_{0}}{\left(J_{\Theta} J_{\xi}\right)^{5 / 3}}\left(\lambda^{2}-\frac{J_{\Theta} J_{\xi}}{\lambda}\right)+\frac{2}{3} \frac{\mu_{v}}{J_{\Theta} J_{\xi}}\left(2 B_{e_{11}}-B_{e_{22}}-\frac{1}{B_{e_{11}} B_{e_{22}}}\right) \\
& -\frac{p}{J_{\Theta} J_{\xi}} \\
\sigma_{22} & =\sigma_{33}=\frac{2}{3} \frac{\mu_{0}}{\left(J_{\Theta} J_{\xi}\right)^{5 / 3}}\left(\frac{J_{\Theta}}{\lambda}-\lambda^{2}\right)+\frac{2}{3} \frac{\mu_{v}}{J_{\Theta} J_{\xi}}\left(2 B_{e_{22}}-B_{e_{11}}-\frac{1}{B_{e_{11}} B_{e_{22}}}\right) \\
& -\frac{p}{J_{\Theta} J_{\xi}}
\end{aligned}
$$




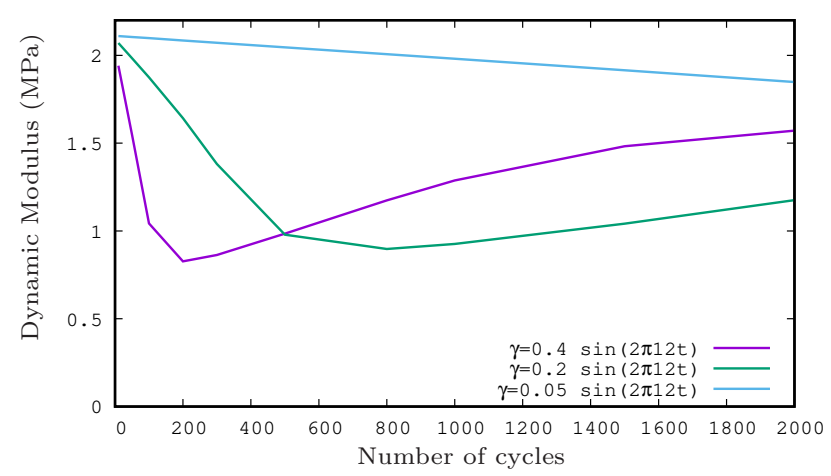

Fig. 6 Dynamic modulus evolution during cyclic simple shear tests for various amplitudes at $f=12 \mathrm{~Hz}$ and $p=0 \mathrm{Mpa}$

As $J_{m}=1$ (incompressible case), eq. (26) does not apply and $p$ is defined from the condition of uniaxial stress $\left(\boldsymbol{\sigma}_{22}=\boldsymbol{\sigma}_{33}=0\right)$ :

$$
p=\frac{2}{3} \frac{\mu_{0}}{\left(J_{\Theta} J_{\xi}\right)^{2 / 3}}\left(\frac{J_{\Theta}}{\lambda}-\lambda^{2}\right)+\frac{2}{3} \mu_{v}\left(2 B_{e_{22}}-B_{e_{11}}-\frac{1}{B_{e_{11}} B_{e_{22}}}\right)
$$

The mechanical and chemical evolutions equations are obtained from eqs. (38) considering $K_{v} \rightarrow \infty$. The thermal equilibrium simplifies to the one defined in the previous example with a supplementary source term $r(t)$ :

$$
\begin{aligned}
& \frac{\rho_{0}}{J_{\Theta} J_{\xi}} C_{p} \dot{\Theta}=l_{m}+l_{\xi}+\phi_{m}+\phi_{\xi}+\frac{\rho_{0}}{J_{\Theta} J_{\xi}} r(t), \quad \Theta(0)=293 K \\
& \text { with } \rho_{0} r(t)=\left\{\begin{array}{l}
28 e^{6}\left(\frac{t}{30}\right) \text { if } t \leq 30 \\
0 \text { if } t>30
\end{array}\right.
\end{aligned}
$$

In figure 7, we show the numerical responses of the same explicit integration scheme as used in the previous example. One can remark that the higher the extension (or compression) is, the lower the chemical conversion is. In figures 7 (c) and $7(\mathrm{~d})$, the thermal aging effect on the relaxed component of the stress is illustrated.

\section{Conclusion}

The thermo-chemo-mechanical coupling in soft materials such as rubbers or polymers is of high interest to understand and modelize the complex interactions that take place in these materials when cyclic thermo-mechanical loadings are considered. In this paper, we propose a general constitutive multiphysics model that can phenomenologically represent this complex behavior. We show, on trial examples, that this framework can qualitatively represent thermo-mechanical aging for incompressible and nearly incompressible materials. We adopt a mixed free energy form, that can be linked to a partial Legendre transform of the Helmoltz free energy. Both the mixed form and the splitting of the volume variation into mechanical, chemical and thermal contributions allow to naturally introduce a 


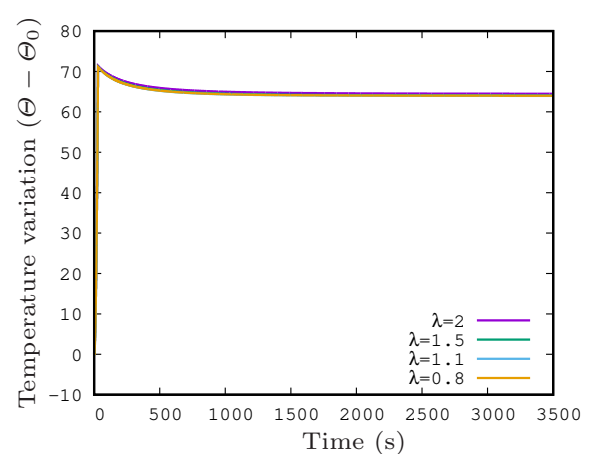

(a) Temperature variation

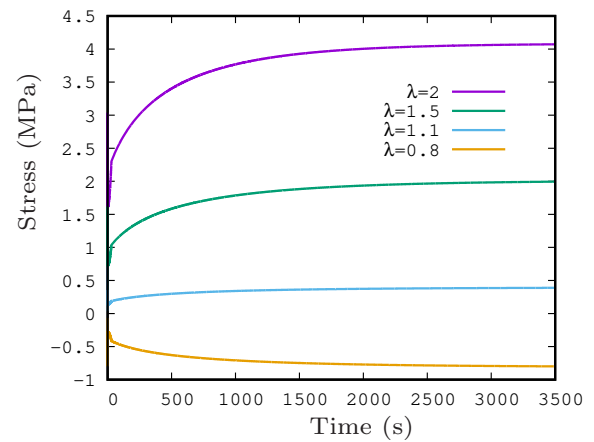

(c) Uniaxial stress

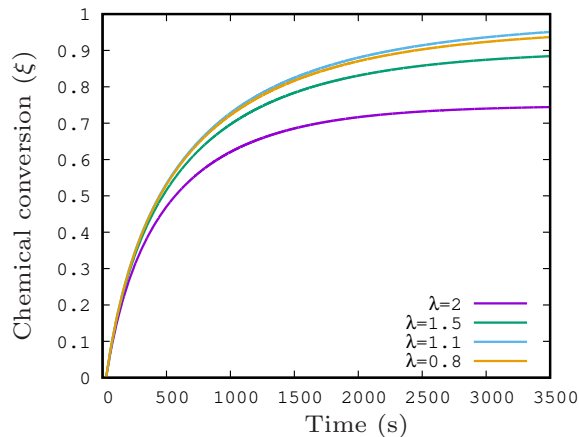

(b) Chemical conversion

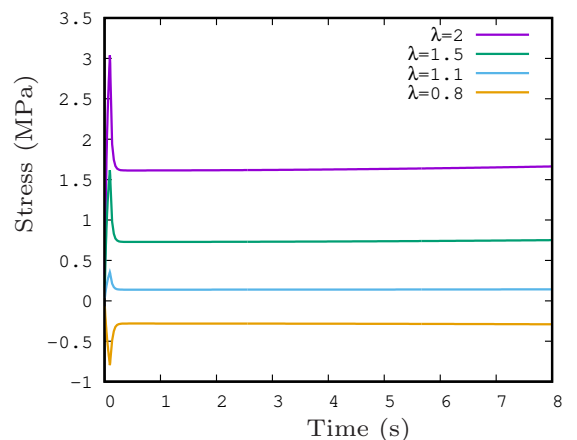

(d) Uniaxial stress (zoom in the firt seconds)

Fig. 7 Results of the thermal aging uniaxtial tension test for various values of extension in adiabatic conditions

pressure dependency of the chemical evolution law.

The introduction of the proposed model in Finite-Elements is straightforward using a monolithic multi-fields approach. This step is required to identify the material parameters and to fully validate the model. From an experimental point of view, it is mandatory to consider non homogeneous tests as it seems impossible to control and measure locally a chemical conversion during a thermo-mechanical test. Furthermore, chemo-physical aging could also be coupled to other physical phenomena like damage, strain induced crystallization and so on. It is therefore required to provide new and original, experimental tests or experimental methods to investigate these coupled phenomena.

\section{References}

André M, Wriggers P (2005) Thermo-mechanical behaviour of rubber materials during vulcanization. International Journal of Solids and Structures 42(1617):4758 - 4778, DOI 10.1016/j.ijsolstr.2005.01.015, URL http://www.sciencedirect.com/science/article/pii/S0020768305000363 
Delattre A, Lejeunes S, Lacroix F, Mo S (2016) On the dynamical behavior of filled rubbers at different temperatures: Experimental characterization and constitutive modeling. International Journal of Solids and Structures 90:178 - 193, DOI http://dx.doi.org/10.1016/j.ijsolstr.2016.03.010, URL http://www.sciencedirect.com/science/article/pii/S0020768316001293

Garnier P, Cam JBL, Grdiac M (2013) The influence of cyclic loading conditions on the viscoelastic properties of filled rubber. Mechanics of Materials 56:84 - 94, DOI http://dx.doi.org/10.1016/j.mechmat.2012.10.001, URL http://www.sciencedirect.com/science/article/pii/S0167663612001688

Gigliotti M, Grandidier JC (2010) Chemo-mechanics couplings in polymer matrix materials exposed to thermo-oxidative environments. Comptes Rendus Mcanique 338(3):164 - 175, DOI http://dx.doi.org/10.1016/j.crme.2010.02.008, URL http://www.sciencedirect.com/science/article/pii/S1631072110000240

Gigliotti M, Grandidier JC, Lafarie-Frenot MC (2011) Assessment of chemo-mechanical couplings in polymer matrix materials exposed to thermo-oxidative environments at high temperatures and under tensile loadings. Mechanics of Materials 43(8):431 - 443, DOI http://dx.doi.org/10.1016/j.mechmat.2011.04.007, URL http://www.sciencedirect.com/science/article/pii/S016766361100069X

Grandcoin J, Boukamel A, Lejeunes S (2014) A micro-mechanically based continuum damage model for fatigue life prediction of filled rubbers. International Journal of Solids and Structures 51(6):1274 - 1286, DOI http://dx.doi.org/10.1016/j.ijsolstr.2013.12.018, URL http://www.sciencedirect.com/science/article/pii/S0020768313004915

Johlitz M, Lion A (2013) Chemo-thermomechanical ageing of elastomers based on multiphase continuum mechanics. Continuum Mechanics and Thermodynamics 25(5):605-624, DOI 10.1007/s00161-012-0255-8, URL http://dx.doi.org/10.1007/s00161-012-0255-8

Kannan K, Rajagopal K (2011) A thermodynamical framework for chemically reacting systems. Zeitschrift fr Angewandte Mathematik und Physik (ZAMP) 62:331-363, URL http://dx.doi.org/10.1007/s00033-010-0104-1, 10.1007/s00033-010-0104-1

Lion A, Höfer P (2007) On the phenomenological representation of curing phenomena in continuum mechanics. Arch Mech 59(1):59-89

Lion A, Dippel B, Liebl C (2014) Thermomechanical material modelling based on a hybrid free energy density depending on pressure, isochoric deformation and temperature. International Journal of Solids and Structures 51(34):729 - 739, DOI http://dx.doi.org/10.1016/j.ijsolstr.2013.10.036, URL http://www.sciencedirect.com/science/article/pii/S0020768313004319

Loeffel K, Anand L (2011) A chemo-thermo-mechanically coupled theory for elasticviscoplastic deformation, diffusion, and volumetric swelling due to a chemical reaction. International Journal of Plasticity 27(9):1409 - 1431, DOI 10.1016/j.ijplas.2011.04.001, URL http://www.sciencedirect.com/science/article/pii/S0749641911000507

Mahnken R (2013) Thermodynamic consistent modeling of polymer curing coupled to viscoelasticity at large strains. International Journal of Solids and Structures 50(13):2003 - 2021, DOI http://dx.doi.org/10.1016/j.ijsolstr.2013.01.033, URL http://www.sciencedirect.com/science/article/pii/S0020768313000486

NGuyen T, Lejeunes S, Eyheramendy D, Boukamel A (2016) A thermodynamical framework for the thermo-chemo-mechanical couplings in soft materials at finite strain. Mechanics of Materials 95:158 - 171, DOI http://dx.doi.org/10.1016/j.mechmat.2016.01.008, URL http://www.sciencedirect.com/science/article/pii/S016766361600017X

Prigogine I (1968) Introduction to thermodynamics of irreversible processes. third edition, Interscience Publishers 